\title{
LES TRAVAUX DE LA PLAINE DES BENI-MOUSSA
}

(Maroc)

\section{I. - EXPOSÉ GÉNÉRAL ET CONSISTANCE DES TRAVAUX}

La France, fidèle à sa mission et à son génie créateur, poursuit, depuis de longues années, dans les états associés, et souvent en dépit de nombreuses difficultés économiques, un plan d'équipement ration-
L'objet de notre propos sera précisément la description d'une partie de cet ensemble: les travaux qui viennent d'être réclisés doms la plaine des BéniMoussa et qui répondent à la double exigence de

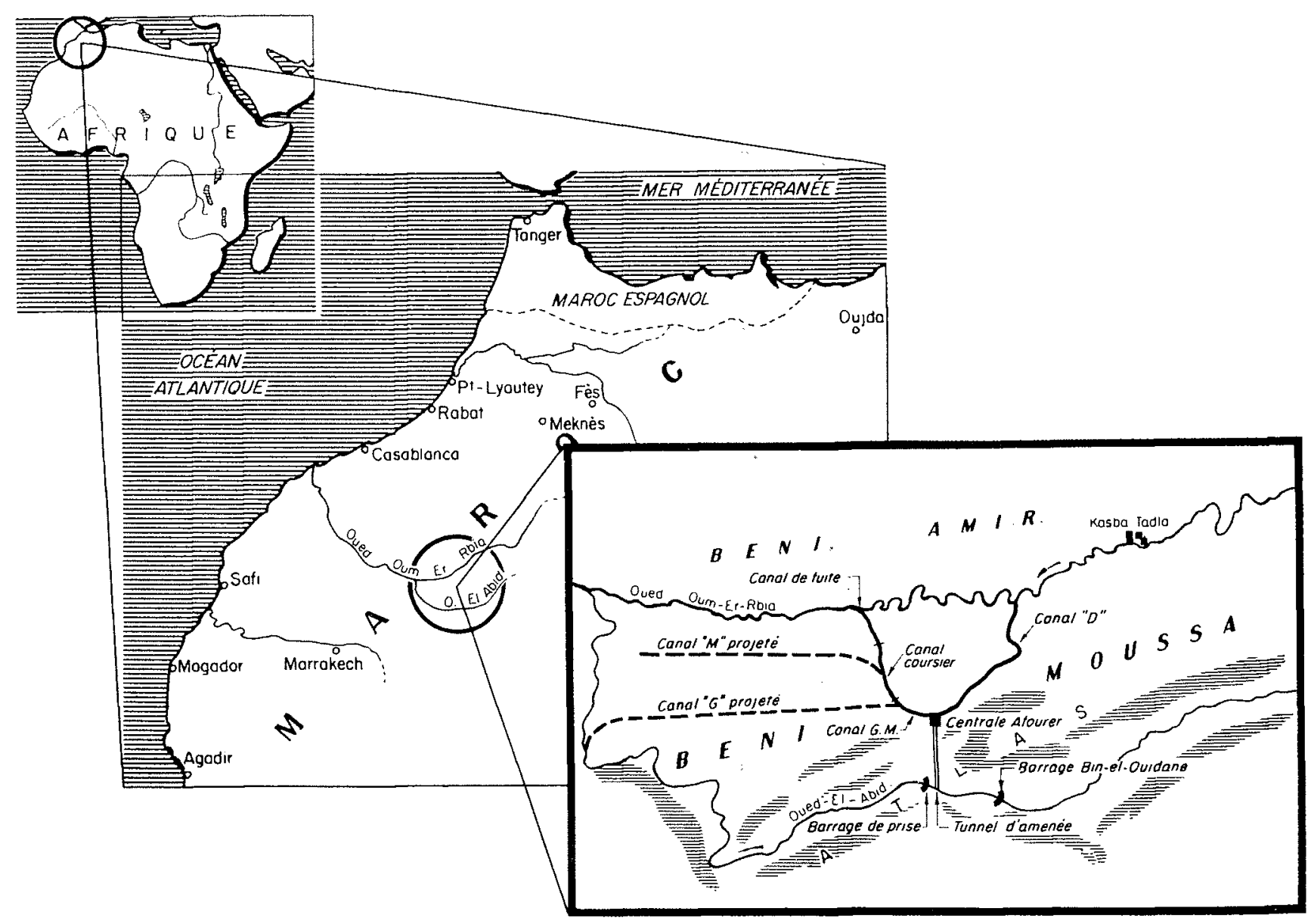

nel et grandiose. Se plaçant à la fois au point de vue industriel, agricole, médical, sanitcire et culturel, elle cherche, par des initiatives hardies et incessantes, d̀ en faire des pays modernes, au niveau de vie plus élevé, oì le mieux-être s'accentue d'onnée en année. Elle a créé tour à tour des instituts de recherches scientifiques spécialisés, des hôpitaux, des routes, des ports, des barrages, des comaux d'irrigation et de drainago, cherchant constamment, en outre, à améliorer l'habitat.

Elle est particulièrement fière de l'ensemble de ses réalisations marocaines. l'agriculture et de la jeune industrie marocaines.

Ils consistent essentiellement dans la centrale hydroélectrique d'Afourer et dans les canaux primaires d'irrigation de la plaine des Béni-Moussa.

Disons d'abord quelques mots du cadre géographique dans lequel s'inscrivent ces travaux. La plaine du Tadla, située cu pied du Moyen Atlas, est divisée par l'Oued Oum-er-Rbia en deux parties portant les noms de deux tribus: les. Béni-Amir sur la rive droite et les Béni-Moussa sur la rive gauche.

L'Oum-er-Rbic a pour principal affluent l'Oued El 
Abid, dont la partie moyenne, sensiblement orientée Est-Ouest, est parallèle à sa vallée et se trouve à environ 250 mètres au-dessus de la plaine des BéniMoussa.

L'industrie marocaine, en pleine expomsion, a des bosoins sans cesse accrus d'énergie. La situation géo- a été construite à Bin-el-Ouidane et la seconde, qui lui fait suite, à Afourer.

La plaine du Tadla, aride en raison de la sécheresse, est cependant très propice à l'agriculture. La partie déjà irriguée des Béni-Amir a répondu à tous les espoirs, et le paysan marocain, fixé sur ces terres auparavoni hostiles, cultive avec succès le blé dur et le blé tendre,

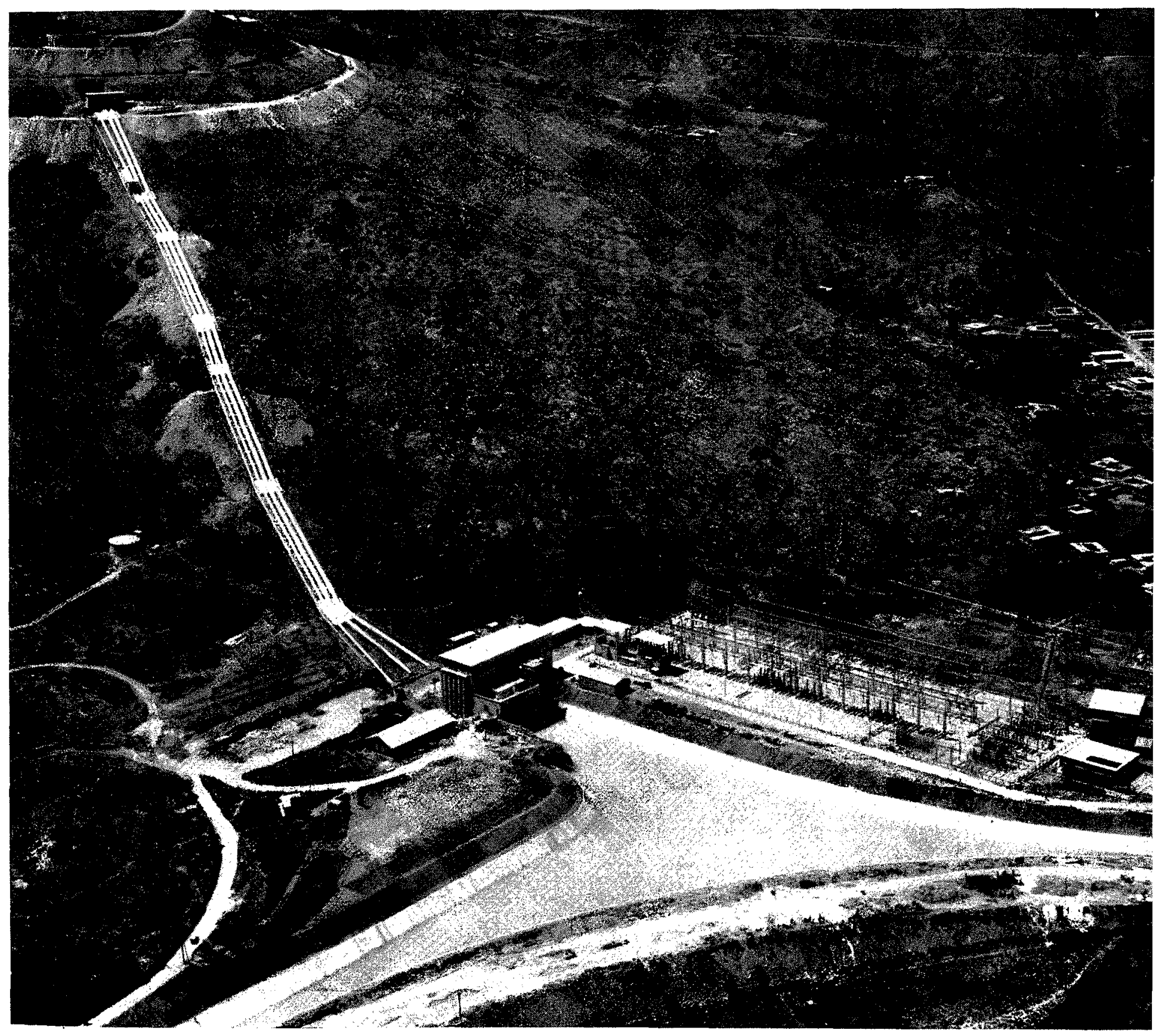

Usine hydroelectrique d'Afourer avec le bassin de compensation, origine des canaux d'irrigation.

graphique de l'Oued Ed Abid a permis la construction de deux centrales hydro-électriques composant l'ensemble le plus important qui ait été réalisé jusqu'ici par la France au Maroc. La première de ces centrales l'orge, la fève, la luzerne, les légumes et les arbres fruitiers.

L'expérience très encourageante des Béni-Amir a donc conduit l'Administration française à réaliser l'irri- 


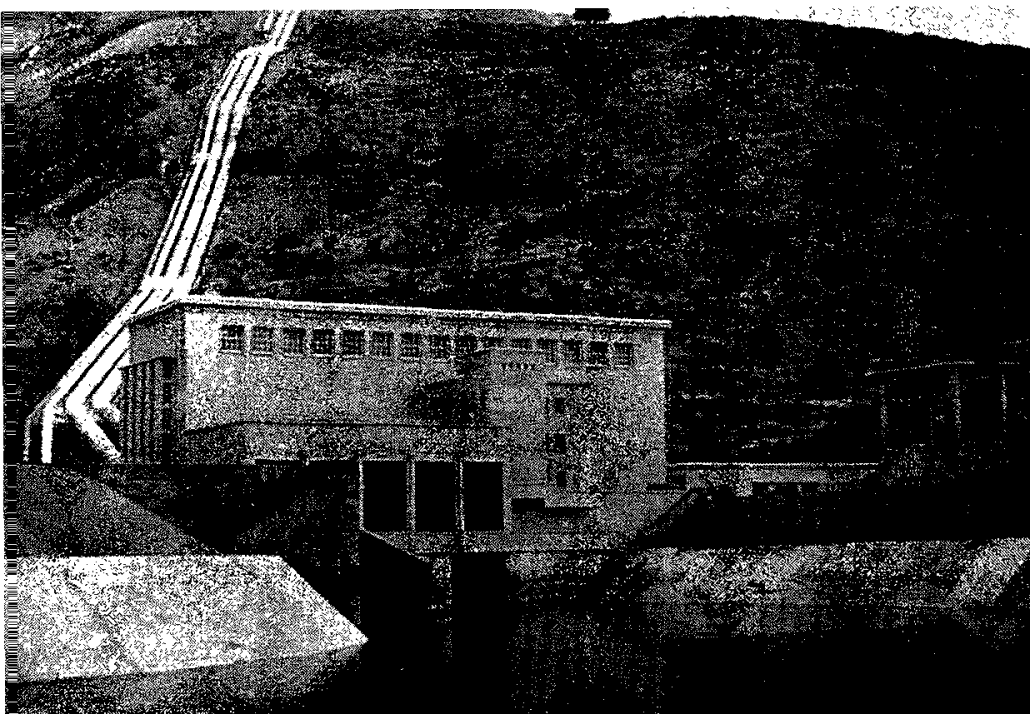

Usine hydroélectrique d'Afourer. gation de la rive gauche de l'Oued Oum-er-Rbia, c'està-dire la plaine des Béni-Moussa

\section{II. - CENTRALE HYDRO-ÉLECTRIQUE D'AFOURER}

\section{a) CONDUITES FORCÉES :}

Les ecux de l'Oued El Abid, "turbinées " une première fois à Bin-el-Ouidane, sont ensuite dirigées, après le barrage de prise d'Ait Ouarda, vers les deux conduites forcées d'Afourer par un tunnel de 10 kilomètres de long.

Ces conduites forcées métalliques, qui ont $2,60 \mathrm{~m}$ de diamètre et $540 \mathrm{~m}$ de longueur unitaire, canalisent la dérivation de l'Oued El Abid limitée volontairement à $46 \mathrm{~m}^{3} / \mathrm{s}$. Une troisième conduite, dite conduite de

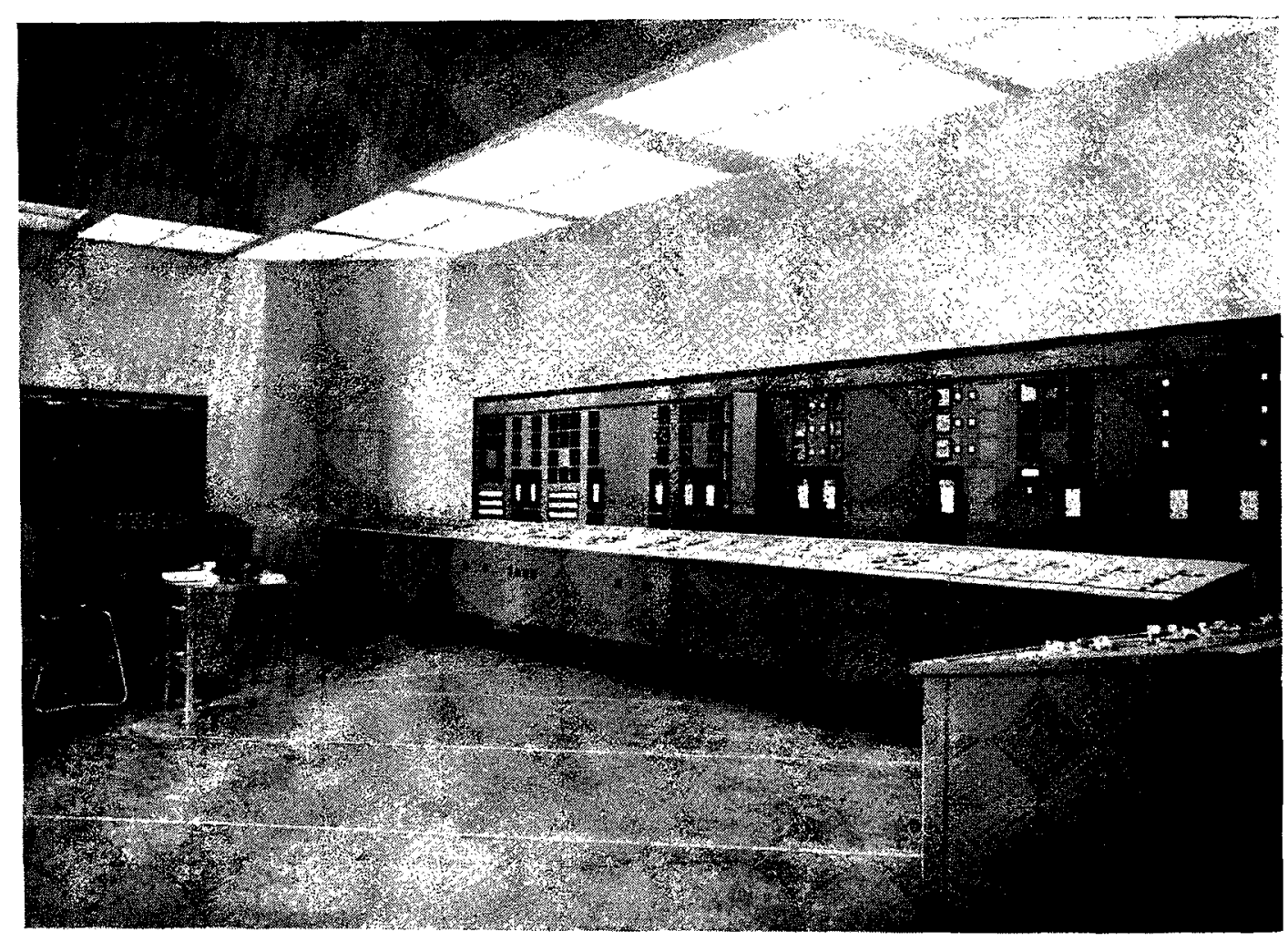

Salle des tableaux

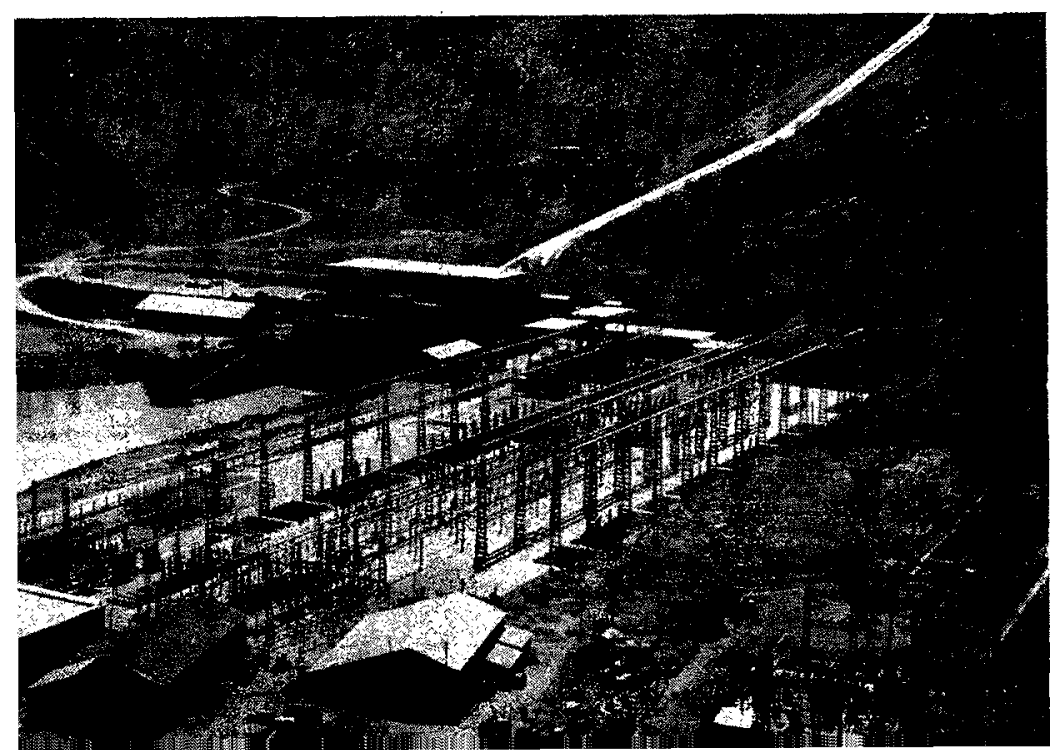

décharge, débitera directement dans le bassin de com. pensation situé immédiatement à l'arval de l'usine. Elle $a$ un diamètre décroissant et permettra l'alimentation des cancux d'irrigation pendant les arrêts de l'usine.

Toutes ces conduites, entièrement soudées, sont du type auto-fretté à frettage souple à enroulement hélicoildal. Elles reposent sur des berceaux en béton et leur stabilité est assurée par huit massifs d'ancrage.

Elles n'ont pu être enterrées en raison des frais considérables de terrassement et de remblaiement. Elles ont donc été pourvues de joints de dilatation et, pour limiter leur échauffement sous l'action du soleil, elles ont éié enduites d'une peinture aluminium.

Les conduites ne comportent pas de collecteur. Elles 
sonl raccordées directement aux vannes sphériques d'entrée aux turbines par l'intermédiaire de cônes.

\section{b) USINE PROPREMENT DITE :}

Elle comprend essentiellement un bâtiment en béton armé de 50 mètres de long, de 18 mètres de large et de 30 mètres de haut doms la salle des machines. Ce bâtiment abrite les deux groupes principaux distants de $13,50 \mathrm{~m}$ d'axe en axe, et constitués chacun par une turbine Francis et un alternateur à axe vertical. La puissance unitáire de ces groupes' s'élève ò $46.000 \mathrm{~kW}$.

En service, les turbines, d'une puissance de $65.000 \mathrm{ch}$ tournent à une vitesse de $427 \mathrm{t} / \mathrm{mn}$ sous une hauteur tuées à l'irrigation. Pour assurer une compensation partielle des débits reçus en aval de l'usine et, en outre, pour éviter la propagation dans les canaux de perturbations résultant de variations de charge, il a été prévu un bassin de compensation d'une superficie de 2 ha, retenont un volume d'eau d'environ $25.000 \mathrm{~m}^{3}$.

\section{III. - CANAUXX D'IRRIGATION DE LA PLAINE DE BENI-MOUSSA}

L'ossature maîtresse du réseau d'irrigation conlprend deux bronches principales desservant, l'une,

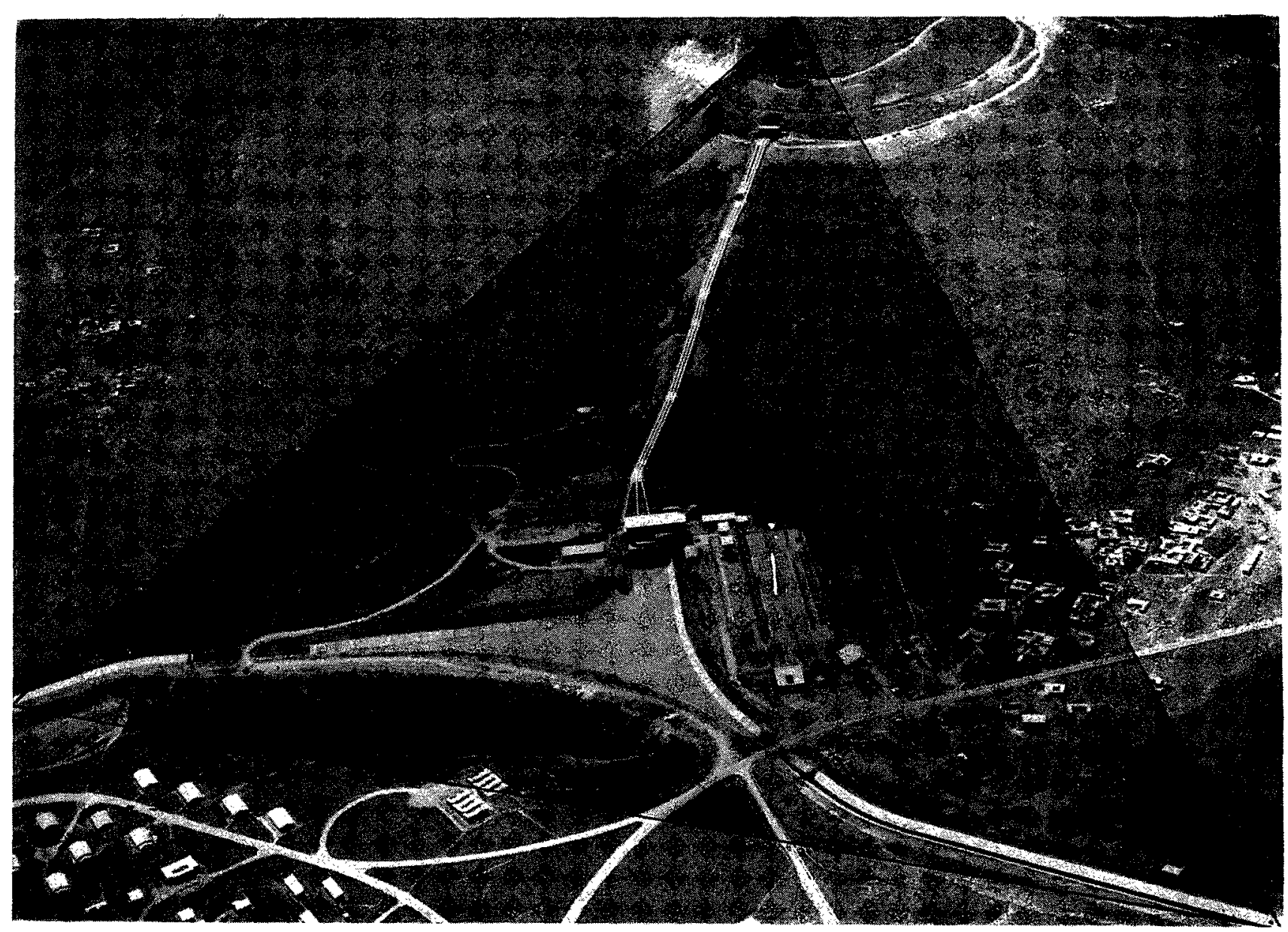

Centrale et départ des canaux.

de chule moyenne de $228 \mathrm{~m}$ et absorbent un débit de $24 \mathrm{~m}^{3} / \mathrm{s}$.

Ce bâtiment abrite, en outre, deux groupes auxiliaires, à cxe horizontal, composés chacun d'une turbine Pelton de $690 \mathrm{ch}$ et d'un alternateur $600 \mathrm{kVA}$, tournant à une vitesse de $750 \mathrm{t} / \mathrm{mn}$ en absorbant un débit de $2.750 \mathrm{l} / \mathrm{mn}$.

La production annuelle de l'usine sera de l'ordre de 350 millions de kilowatts-heure.

\section{c) BASSIN DE COMPENSATION :}

Les eaux " turbinées " à Afourer sont ensuite resti- la partie Est, l'autre la partie Ouest de la plaine des Béni-Moussa. L'alimentation des conaux Est et Ouest se fait aux deux extrémités du bassin de compensation par des vannes à nivecu aval constant.

La branche Est porte le nom de a Canal $D$; il a $33 \mathrm{~km}$ de long, une largeur de 12 à $16 \mathrm{~m}$ entre berges et un débit maximum de $16 \mathrm{~m}^{3} / \mathrm{s}$.

La branche Ouest comprend plusieurs parties. D'abord le "Canal GM », long de $8,400 \mathrm{~km}$, large de $16 \mathrm{~m}$ et profond de $4,50 \mathrm{~m}$. Il est prolongé jusqu'à l'ouvrage de restitution sur l'Oued Oum-er-Rbia par deux nouvecux tronçons: 
- la construction de ponts et de passerelles au-dessus des conaux;

- la construction de cités, bâties en moellons, avec douches et réfrigérateurs, pour cadres et ouvrie:s des chantiers;

- la construction de deux villages, avec souks ef mosquée, pour le recasement des cultivateurs marocains expropriés avant la mise en eau du barrage de Bin-el-Ouidane;

- la construction de la centrale d'Afourer avec ses dépendances.

Le creusement des canaux s'est effectué au moyen de pelles mécaniques Koehring équipées en draglines. L'entreprise disposait sur le chantier de 3 pelles 605 de 1.200 litres de capacité de godet et de 3 pelles 304 de 750 litres. Ces engins ont reçu différents équipements suivont les nécessités du moment.

Au cours de ces travaux de terrassement, on a rencontré des croûtes calcaires plus ou moins épaisseg qu'il a fallu désagréger à l'explosif. L'air comprimé nécessaire cux marteaux pneumatiques, pour la perforation des trous de mine, était fourni par 5 compresseurs HK 500 Ingersoll Rand, 3 compresseurs Flottmann et 5 compresseurs Broomwade.

Après le creusement des canaux et partout où ces derniers n'étaient pas entièrement en déblai, des digues en terre compactée ont été établies pour conserver aux canaux le profil reconnu nécessaire. Ces digues ont une épaisseur de $3 \mathrm{~m}$ et de $9 \mathrm{~m}$.
Pour les travaux d'établissement des digues, l'entreprise disposait de 6 tracteurs D8 Caterpillar de 150 cil, de 10 roulecuux pied de mouton Le Toumeau, de 6 citernes à eau et de 2 niveleuses Caterpillor.

Le bâtiment de l'usine ef le bassin de compensation, situés cu pied de l'Atlas, ont nécessité l'extraction d'un cube important de rocher. Ces travaux ont été exécutés par des méthodes classiques.

\section{承}

Le revêtement des canaux présente beaucoup d'intérêt car il a été exécuté à l'aide de machines spécialement conçues pour ce travail.

Deux trains de machines ont été utilisés, chaque train comprenant:

$$
\begin{aligned}
& \text { - une taluteuse, } \\
& \text { - une bétonneuse, } \\
& \text { - une coupeuse de joints, } \\
& \text { - une finisseuse. }
\end{aligned}
$$

Chaque machine est constituée d'une ossature métallique épousont le profil du canal à revêtir et est supportée par des châssis formant boogie. Une passerelle, située d̀ la portie supérieure, porte tous les appareils nécesscires à son fonctionnement. Sur chaque berge est posée une voie ferrée sur laquelle progressent simultanément les quatre machines.

Un dispositif spécial règle automatiquement, à quel- 


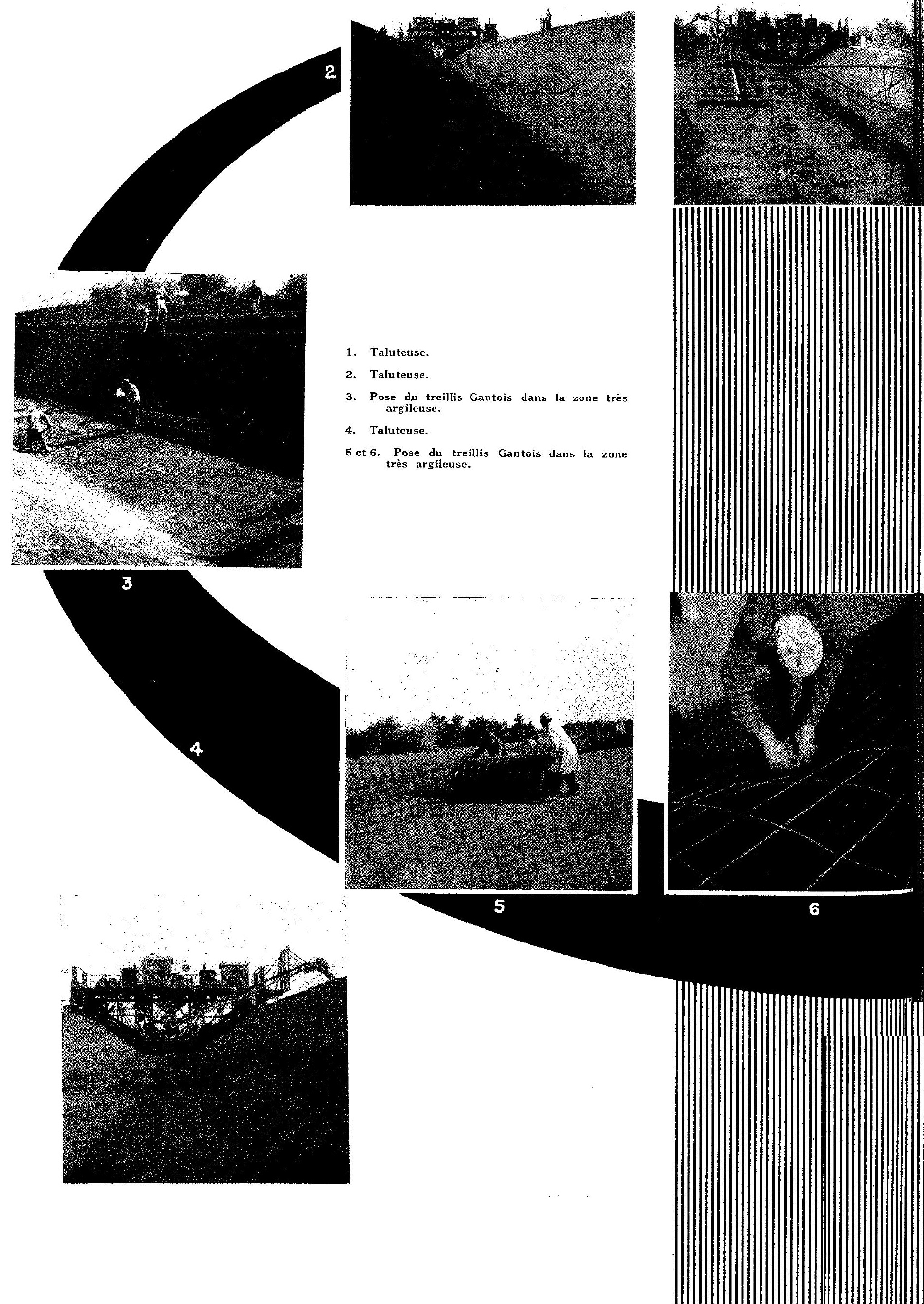




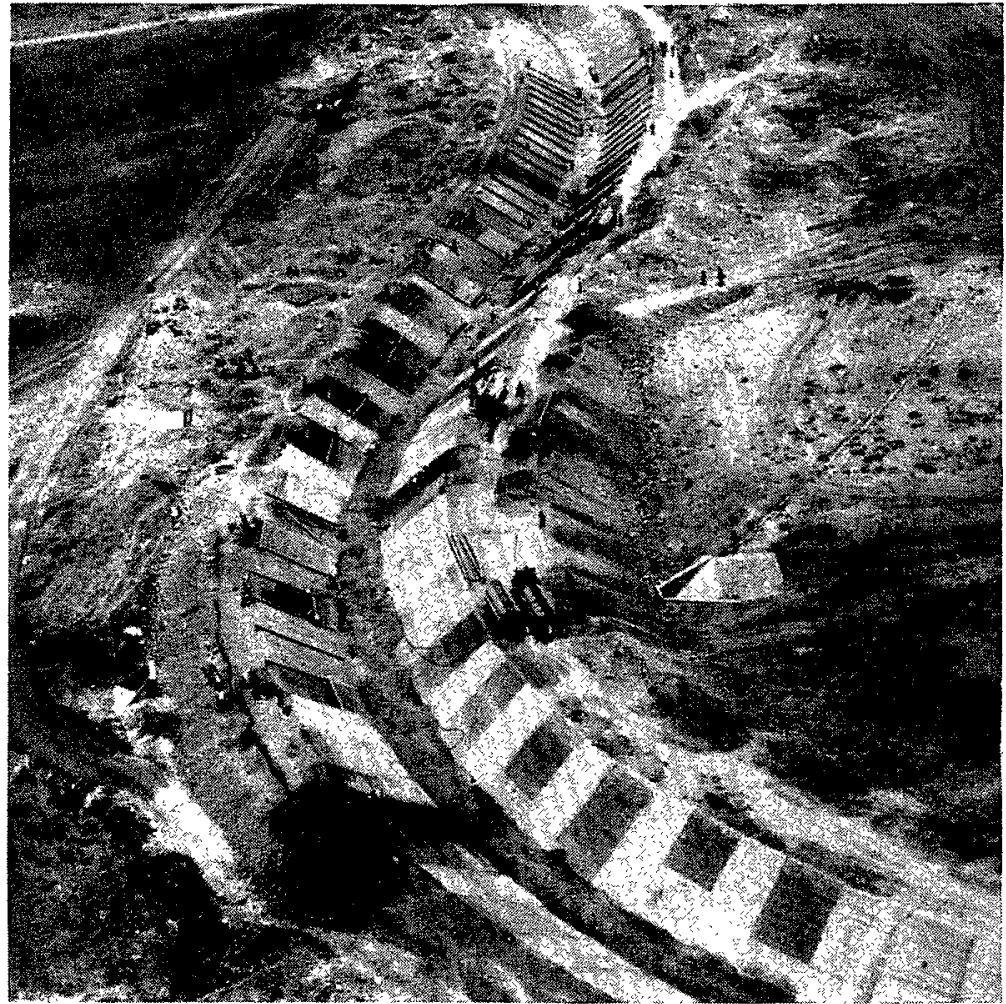

ques millimètres près, le nivellement ef le bétonnage.

Le premier engin est la taluteuse qui règle les parois.

Derrière la taluteuse vient la bétonneuse. Cette machine est munie de goulottes, réparties sur tout le profil, et d'un dispositif de lissage du béton. Un système de couteaux ménage les joints longitudi. naux qui seront ensuite parfaits à la main.

Le béton est préparé dans une bétonnière mobile sur chenilles, du type $34 \mathrm{E}$ Koehring, à double tambour et munie d'un bras orientable sur lequel se déplace un godet.

La coupeuse de joints, qui porte à sa partie inférieure des coutecux à lame vibrante pouvont être montés ou descendus, sert à découper les joints transversaux.

Enfin, la finisseuse, dernier élément du train, est une sorte de pont, réglable dans le sens vertical au moyen de vérins montés sur boogies, et qui porte tous les ap. pareils destinés à la finition complète de la dalle. C'est de la finisseuse que l'on applique le "mastic filler " ainsi que le " curing compound " pulvérisé sur toute

Bétonneuse vue de l'avant. Train de machines.

Chantier de corroyage.

sur le canal G.M. (section rocheuse).

Canal coursier :

Au 1 er plan, la taluteuse;

au $2^{\mathrm{e}}$ plan, les autres machines.

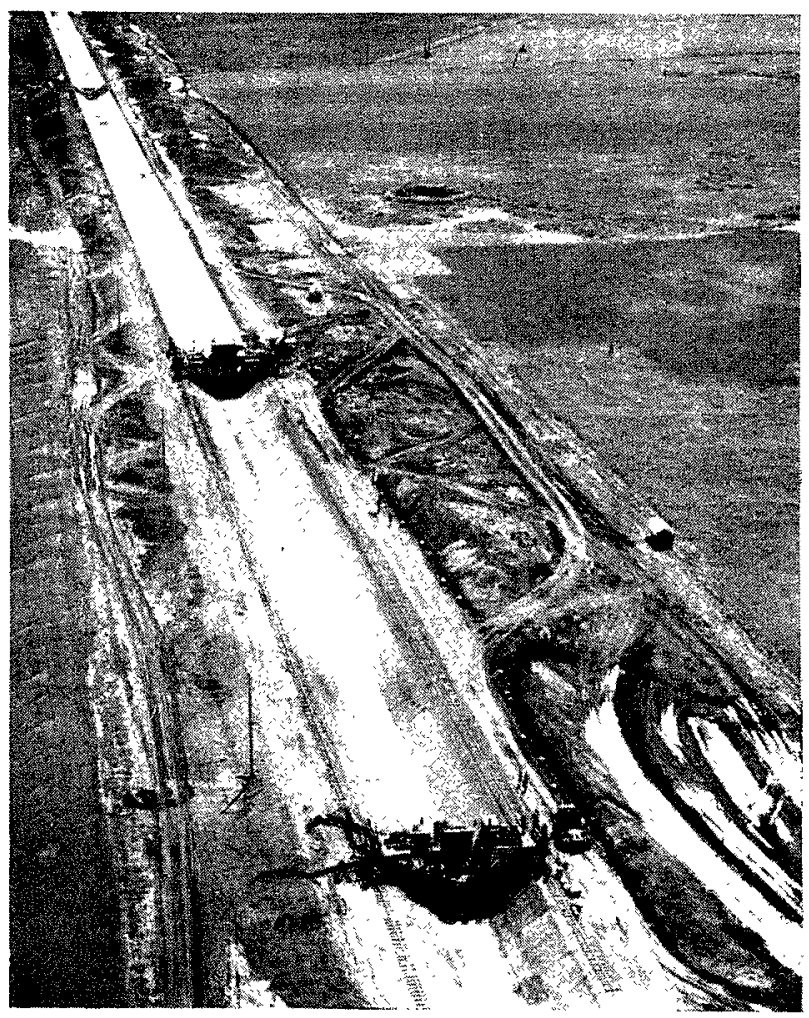

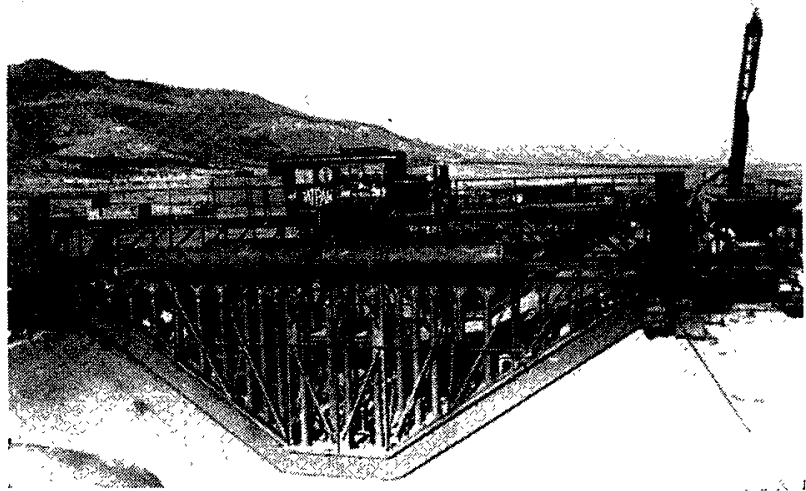

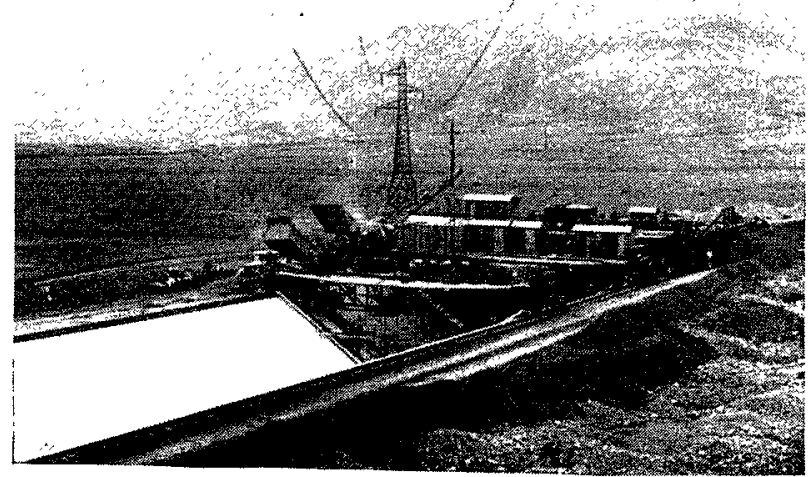




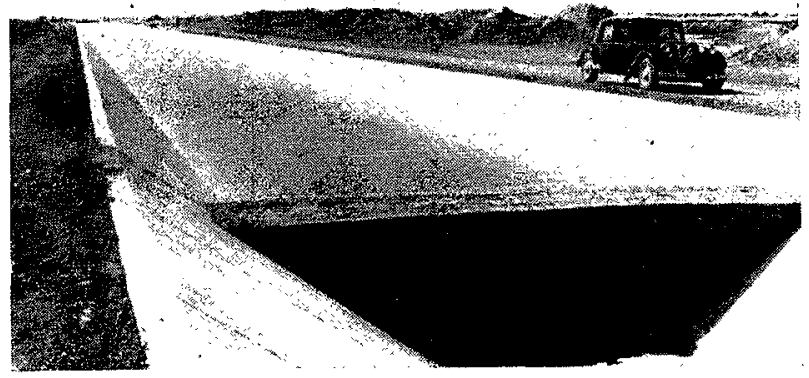

Batardeau de mise en eau provisoire pour les mesures d'étanchéité.

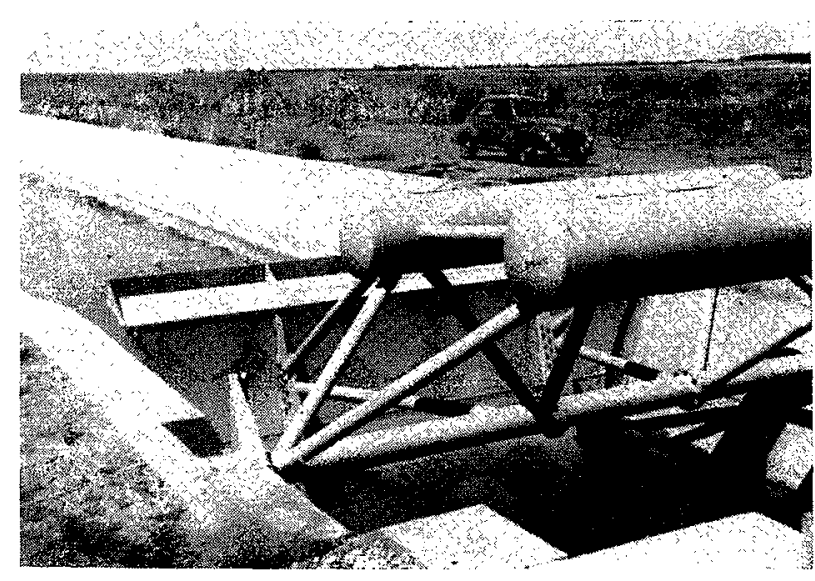

Vannes Neyrpic.

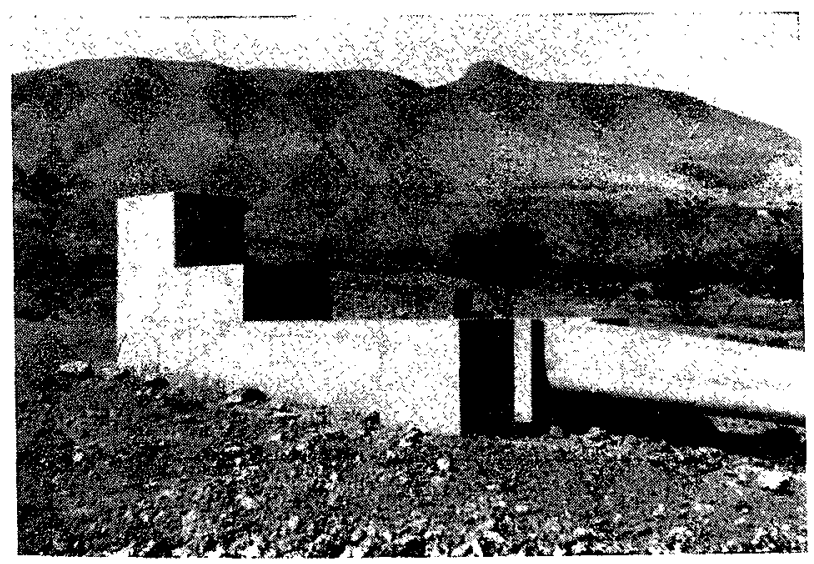

Prise secondaire. la surface du béton et dont l'effet est d'empêchér toute évaporation de l'eau de prise.

Les 4 machines sont automotrices.

Indépendarmment des 2 trains de machines et des 2 pavers $34 \mathrm{E}$ Koehring, le chantier de revêlement disposait de 12 camions de 8 à 25 tonnes, de 6 camions de 4 tonnes, de 6 tracteurs "Autocar" et de voitures légères.

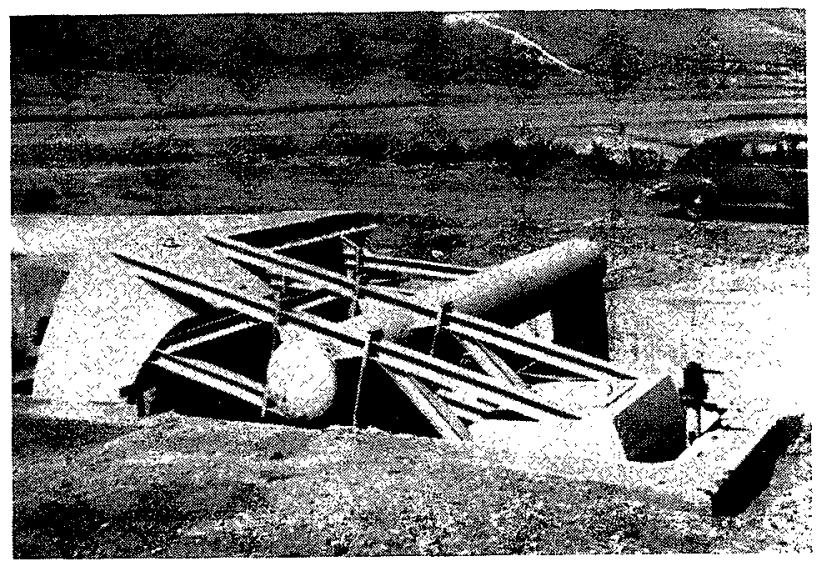

Vannes Neyrpic.

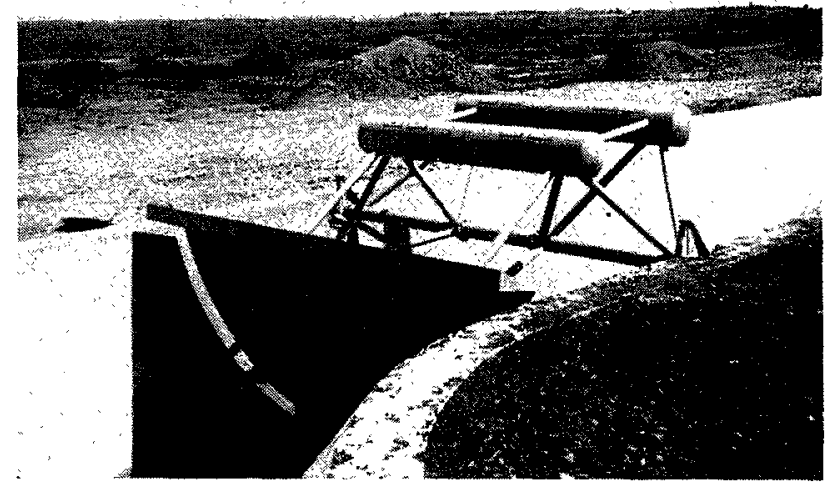

Vannes Neyrpic.

Deux stations de dosage et une installation de concassage-criblage-lavage complétaient l'équipement.

:

Sur certaines parties de leur parcours, les canaux ont dû, nous l'avons vu, être exécutés en bélon armé. Or, pour ne pas ralentir la vitesse d'avancement des machines qui exécutaient le revêtement, on retint le principe d'armatures soudées, dont la mise en place est très rapide et qui permettent d'éviter l'exécution d'un nombre très important de ligatures.

Le choix se porta sur un grillage soudé Gantois, de maille $250 \times 250$ et constitué par du fil d'acier de $3.9 \mathrm{~mm}$. Présentée sous forme de rouleaux triples de 
$75 \mathrm{~m}$ de longueur, celte armature était débitée sur place en panneaux correspondant à la longueur d'une section de canal, et mise en place par une main-d'œuvre réduite.
L'avancement horaire du travail fut ainsi en moyenne de $30 \mathrm{~m}$ et l'emploi des $13.000 \mathrm{~m}^{2}$ d'armatures soudées permit de faire l'économie de 325.000 ligatures.

\section{V. - CONCLUSION}

La plaine des Béni-Moussa semble plus fertile encore que celle des Béni-Amir. L'irrigation transformera
Et c'est ainsi que chaque année voit la réalisation d'une partie ou d'une autre du vaste plan d'équipe-

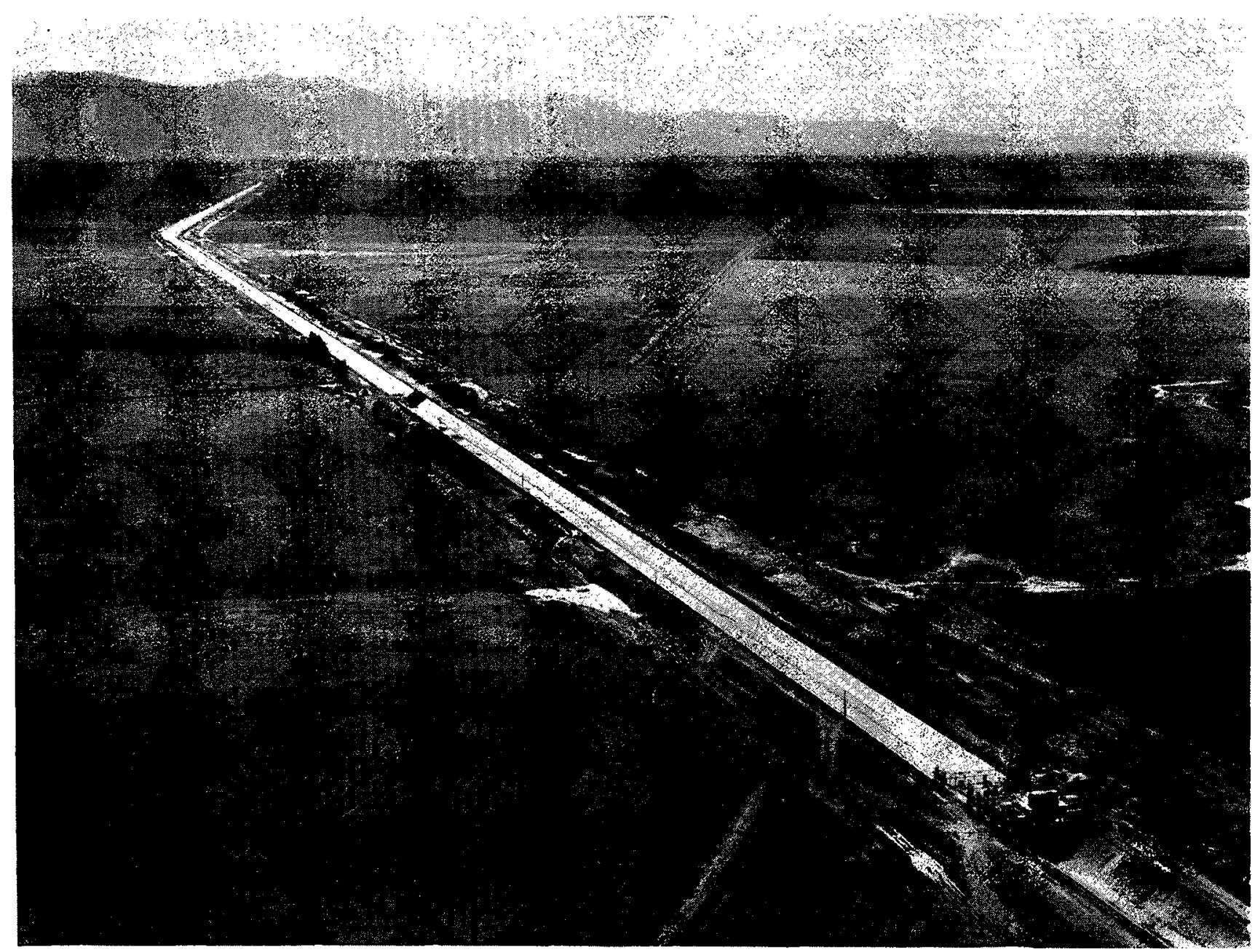

Canal coursier en cours de bétonnage.

en peu de temps la physionomie de cette région brûléa par le vent. Indépendamment du blé, de l'orge, du maïs et des légumineuses, il est certain que de riches cultures pourront y être faites, en particulier celle des agrumes. Sur cette terre déshéritée, d'une superficie d'environ 100.000 ha, l'eau aura créé la richesse.

Par ailleurs, les lignes à haute tension apporteront à l'industrie des grands centres l'énergie électrique qui est cussi une inappréciable source de mieux-être. ment conçu par la France. Celle-ci sait fort bien d'ailleurs que ce plan n'est pour elle qu'un ordre d'urgence, que sa tâche ne sera pas terminée, et qu'elle continuera d̀ faire bénéficier les états associés de sa culture et des applications bienfaisantes de la science.

\section{A. LARUELLE,}

Société anonyme Ossude, Paris. 